\title{
Benign Mucous Membrane Pemphigoid
}

National Cancer Institute

\section{Source}

National Cancer Institute. Benign Mucous Membrane Pemphigoid. NCI Thesaurus. Code C34907.

A chronic autoimmune disorder characterized by the development of blisters and ulcers in mucous membranes. It affects most often the gums, eyelids and genital mucosa sites. 